\title{
Effect of Corticosteroid Therapy on Fibrinolysis in Patients With Inflammatory and Non-inflammatory Conditions*
}

\author{
R. CHAKRABARTI, M.B. ; G. R. FEARNLEY, M.D., F.R.C.P. \\ ELIZABETH D. HOCKING, M.B., M.R.C.P.ED.
}

Brit. med. F., 1964, 1, 534-537

Fibrin forms a scaffolding for the inflammatory reaction and a barrier to the spread of micro-organisms and irritants. With healing the fibrin is removed, presumably by fibrinolysis. Failure of this to happen accompanies delayed resolution, as in the unresolved pneumonias of pre-antibiotic days. Persistence of fibrin and the formation of fibrinoid are features of chronic inflammation, especially in conditions of unknown causation like rheumatoid arthritis. Whether these features depend on inhibited or inadequate fibrinolysis is unknown but seems a pathogenic possibility.

Corticosteroids have been shown to reduce the plasma fibrinogen level in patients with rheumatic fever (Fearnley, 1951a) and in healthy people (Fearnley and Bunim, 1951), and testosterone has been found to increase the fibrinolytic activity of the blood (Fearnley and Chakrabarti, 1962), indicating that the latter is susceptible to endocrine influence. These observations, together with the finding of low blood fibrinolytic activity in a higher proportion of patients with rheumatoid arthritis than in healthy controls (see below), prompted us to investigate whether treatment with corticosteroids affects fibrinolysis in patients with inflammatory conditions; and, when it became apparent that it does, in patients with non-inflammatory conditions also. We here report that corticosteroids increase the fibrinolytic activity of the blood and discuss the relevance this finding may have to their therapeutic actions.

\section{Material and Methods}

In a pilot study of 50 out-patients with rheumatoid arthritis a single determination of blood fibrinolytic activity was made between 11 a.m. and 12 noon.

The effects of corticosteroids on fibrinolysis, the plasma fibrinogen level, and the blood sedimentation rate were studied in 17 in-patients (12 with inflammatory and 5 with non-inflammatory conditions). Of the patients with inflammatory disease, five received A.C.T.H. gel and seven received prednisone. Of the patients with non-inflammatory disease three were given prednisone, one A.C.T.H. gel, and one cortisone.

The procedure was that used by Fearnley and Chakrabarti (1962) to evaluate the effect of testosterone on fibrinolysis, the blood samples being obtained in the fasting, resting state at 8.30 a.m. After control measurements had been made for several days the treatment was started and the observations were continued. In all but two patients (Cases 1 and 13), in whom less frequent estimations were made, blood fibrinolytic activity was measured daily (except Sunday). The E.S.R. and plasma fibrinogen were measured weekly.

Blood fibrinolytic activity was determined by the dilute blood-clot lysis-time method of Fearnley, Balmforth, and Fearnley (1957), with recording of the end-point as modified by Fearnley and Chakrabarti (1962). The test, set up in

* From the Gloucestershire Royal Hospital, Gloucester. duplicate, consists of the time required for lysis at $37^{\circ} \mathrm{C}$. of clots made with thrombin of a 1:10 dilution of blood in phosphate buffer, $p H$ 7.4. The end-point is recorded visually or photographically, depending on the length of the lysis time. Results are given as lysis-times which are inversely related to blood fibrinolytic activity; hence decrease of lysis-time signifies increase of blood fibrinolytic activity and vice versa. In most of the patients the fibrinolytic response is shown by comparing the mean lysis-time during the control period with that of the last four observations, irrespective of any alteration in dosage. In two patients, however (Cases 10 and 16), in whom reduction of dose caused a marked alteration in fibrinolysis, the mean lysis-time during the last four observations on the initial dosage is used for comparison.

Plasma fibrinogen was estimated gravimetrically by a modification of Fearnley's (1951b) method. The blood sedimentation rate was measured on citrated blood in Westergren tubes.

\section{Pilot Study of Fibrinolytic Activity in Rheumatoid Arthritis}

Table I shows the fibrinolytic activity of 50 out-patients with rheumatoid arthritis (18 men, 32 women), compared with 100 healthy controls (50 of each sex) of approximately similar ages. The latter, whose blood samples were obtained at the same time of day as those of the rheumatoid patients, had served as the controls in a study of fibrinolysis in diabetes mellitus (Fearnley, Chakrabarti, and Avis, 1963). It can be seen that the mean lysis-times of the arthritic patients of both sexes are greater than those of the controls, and that the incidence of low fibrinolytic activity (lysis-time $\geqslant 7$ hours) was higher among the patients with rheumatoid arthritis, especially the males, $50 \%$ of whom had low fibrinolytic activity compared with $10 \%$ of the male controls. It appears that low fibrinolytic activity is commoner among patients with rheumatoid arthritis than among healthy people.

\begin{tabular}{|c|c|c|c|c|}
\hline & \multicolumn{2}{|c|}{ Controls } & \multicolumn{2}{|c|}{ Rheumatoid Arthritis } \\
\hline & Male & Female & Male & Female \\
\hline $\begin{array}{l}\text { Number } \quad . \quad \\
\text { Lysis-time } \geqslant 7 \text { hours } \quad \because \\
\text { Mean lysis-times (hours) }\end{array}$ & $\begin{array}{l}50 \\
5(10 \%) \\
4.5\end{array}$ & $\begin{array}{l}50 \\
7(14 \%) \\
5 \cdot 2\end{array}$ & $\begin{array}{l}18 \\
9(50 \%) \\
6.7\end{array}$ & $10 \stackrel{32}{(31 \%)}$ \\
\hline
\end{tabular}

\section{Effect of A.C.T.H. on Fibrinolysis in Inflammatory Conditions}

A.C.T.H. in gel form was given by injection twice daily to five patients, three with ulcerative colitis and two with rheumatoid arthritis. Table II (Cases 1-5) summarizes the results. It can be seen that all patients showed a pronounced increase of fibrinolytic activity, which was manifest 24 hours after starting treatment in three patients (Cases 2, 3, and 4) 
TABLE II.-Effect of Corticosteroids on Fibrinolysis in Inflammatory and Non-inflammatory Disease

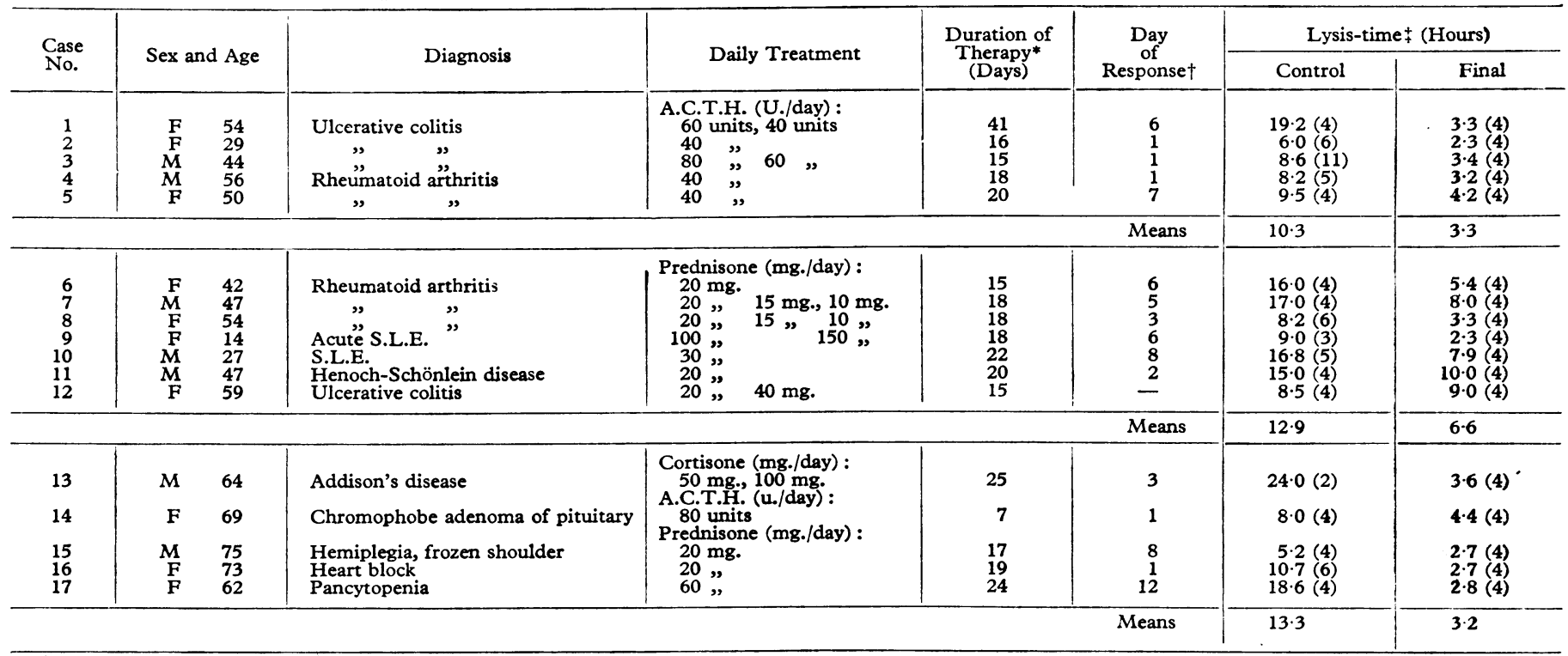

* Duration of observations after treatment was started. † First day after starting treatment on which a significant fall in lysis-time occurred. $\ddagger$ Mean lysis-time during the control period and during the last four days of observation; the figures in parentheses show the number of observations from which the means are derived.

and was apparent on the sixth day in Case 1 and the seventh day in Case 5 . In the three patients who showed a rapid response in respect of fibrinolysis this preceded by several days any evidence of clinical improvement in their condition. Plasma fibrinogen levels and erythrocyte sedimentation rates fell as expected in all of these patients.

Fig. 1 shows the response of fibrinolytic activity in a woman with severe ulcerative colitis (Case 1) whose lysis-times during the control period were long (14-20 hours), fell steeply during treatment, and were maintained at a low level of 2-4 hours.

The mean lysis-time of all patients during the control period was 10.3 hours, and during the last four days of observation 3.3 hours, which indicates a profound effect on blood fibrinolytic activity. In those patients (Cases 1 and 3) in whom the initial dose of A.C.T.H. was subsequently reduced no escape of lysis-time occurred, and indeed all the patients showed a sustained response of fibrinolytic activity during the period of observation.

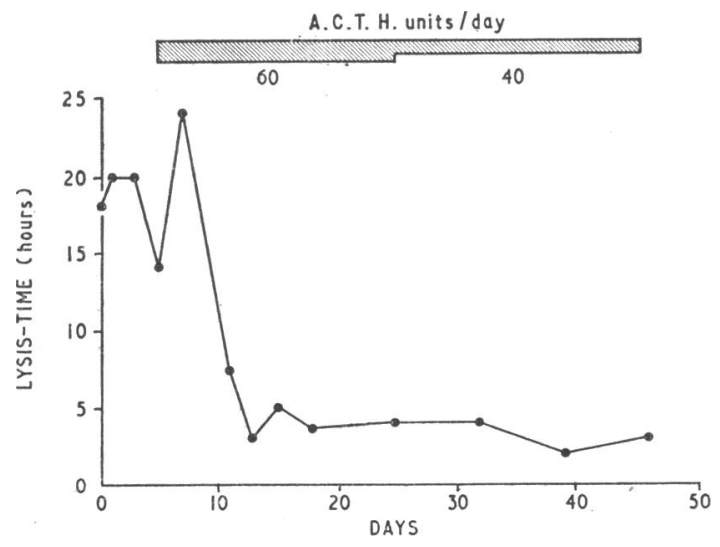

Frg. 1.-Case 1. Reduction of lysis-time (increase of blood fibrinolytic activity) by A.C.T.H. gel in a woman with ulcerative colitis.

\section{Effect of Prednisone on Fibrinolysis in Inflammatory Conditions}

Prednisone in varying dosage was given by mouth to seven patients (Cases 6-12)-three with rheumatoid arthritis, two with systemic lupus erythematosus, one with Henoch-
Schönlein purpura complicated by nephritis, and one with ulcerative colitis. Table II summarizes the results. It can be seen that six of these patients showed an increase of blood fibrinolytic activity but that one (Case 12), a woman with ulcerative colitis, was completely resistant in this respect, her mean lysis-times during the control period and final four days

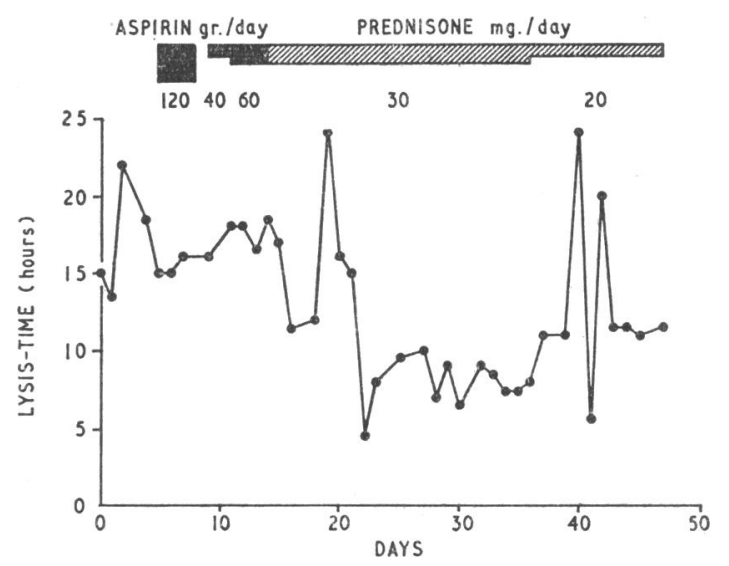

Fig. 2.-Case 10. Effect of prednisone on lysis-time in a man with systemic lupus. Note failure to respond to aspirin and fluctuations of lysis-time when dosage of prednisone was reduced from 30 to $20 \mathrm{mg}$. daily.

of observation being the same. This patient did not improve clinically.

The reduction of lysis-time in these patients appeared to be somewhat less rapid than in those given A.C.T.H., taking two to eight days to become manifest. Furthermore, in some patients the pattern of response was rather different. In Case 11 there was a steep reduction of lysis-time to 5-6 hours lasting for three days, after which the lysis-time rose to 9-11 hours, at which level it remained. In Case 10 (Fig. 2) there appeared to be an initial reduction of lysis time on the second day after starting treatment, a rebound to 24 hours on the fifth day, and a sustained reduction to 7-9 hours thereafter. When the dose of prednisone was reduced from 30 to $20 \mathrm{mg}$. daily there was a swinging escape of lysis-time for several days before it appeared to be settling at a new level of 11 hours. This patient was initially treated with aspirin, which seemed to have no effect on fibrinolysis. 
As with the patients given A.C.T.H., plasma fibrinogen levels and erythrocyte sedimentation rates fell during treatment with prednisone, except in Case 12 . The mean lysistime of all patients during the control period was 12.9 hours, and during the last four days of observation 6.6 hours.

\section{Effect of Corticosteroids on Fibrinolysis in Non-inflammatory Disease}

Conditions neither inflammatory nor malignant which call for corticosteroid therapy are few ; we have been able to make observations in five patients (Table II, Cases 13-17).

Case 13 was that of a man of 64 with hitherto untreated Addison's disease. His clinical condition did not permit more than two control lysis-times, both of which were 24 hours + , before starting treatment with cortisone $50 \mathrm{mg}$. daily, later increased to $100 \mathrm{mg}$. daily. This patient showed a reduction of lysis-time to $7 \frac{1}{2}$ hours on the third day of cortisone therapy, with subsequently a fall to 3-4 hours.

Case 14, a woman of 69 with a chromophobe adenoma of the pituitary, was given a short course of A.C.T.H. gel, 80 units daily, in order to evaluate her adrenal responsiveness, which proved to be normal, as judged by the increase of urinary 17ketogenic steroids. The control lysis-time of 8 hours fell to $4 \frac{1}{2}$ hours.

Case 15, a man of 75 with an old right hemiplegia and frozen shoulder, showed a reduction of lysis-time from a mean of 5.2 hours to 2.7 hours when treated with prednisone, $20 \mathrm{mg}$. daily, as part of an attempt to mobilize his shoulder.

Case 16 was a woman of 73 with disabling symptoms due to a 2:1 atrioventricular block present for three months before admission. Her lysis-times during the control period lay between 10 and 12 hours. Twenty-four hours after starting prednisone, 20 mg. daily, the lysis-time fell to 4 hours and thereafter to $2 \frac{1}{2}-3$ hours. After 19 days' treatment the dose of prednisone was reduced from 20 to $15 \mathrm{mg}$. daily, and this was followed by prolongation of the lysis-time to 6-8 hours for eight days, after which it settled at a level of 4-5 hours. On the fourth day of treatment she was in sinus rhythm, and thereafter lost her symptoms.

Case 17 (Fig. 3) was a woman of 62 suffering from a pancytopenia with hypoplastic marrow. Prednisone was given in a dose of $60 \mathrm{mg}$. daily and the lysis-time initially showed a biphasic

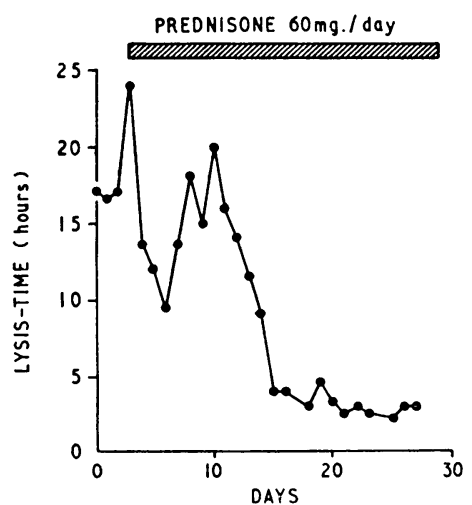

FIG. 3.-Case 17. Reduction of lysistime by prednisone in a woman with pancytopenia. Note delayed biphasic response of blood fibrinolytic activity.

response with reduction on the third day of therapy, followed by prolongation, and then by steep reduction to 3 hours, which was subsequently maintained (see Fig. 3). An excellent haematological response occurred, the haemoglobin rising from $45 \%$ (6.6 g.) to $87 \%(12.7$ g. $)$, the white cells from 1,100 to $4,400 / \mathrm{c} . \mathrm{mm}$., ant the platelets from 98,000 to $272,000 /$ c.mm., but this was delayed until the sustained decrease of lysis-time.

In all of these patients the plasma fibrinogen levels and E.S.R. were within normal limits and in four the former showed a slight fall only during treatment.
The mean lysis-time of all patients during the control period was 13.3 hours, and during the last four days of observation 3.2 hours.

\section{Pattern of Response}

Three patients (Cases 10, 15, and 17) showed a biphasic response of fibrinolytic activity to prednisone consisting of an initial reduction of lysis-time, followed by a rebound escape, and then a further sustained reduction of lysis-time. This pattern, illustrated in Cases 10 (Fig. 2) and 17 (Fig. 3), we have encountered in previous studies of other drugs (Fearnley, Chakrabarti, and Vincent, 1960), and we think it important because it suggests that a pharmacological stimulus to fibrinolysis in some people and perhaps at a critical dosage will provoke a homoeostatic mechanism which can cause a counterswing of fibrinolytic activity.

Perhaps the same mechanism underlay the wide fluctuations of lysis-time shown in Cases 10 (Fig. 2) and 16 when the dose of prednisone was reduced. These fluctuations persisted for several days before the lysis-time settled down to a new level.

\section{Discussion}

The increase of fibrinolytic activity during corticosteroid therapy was of the same order as we have observed in atherosclerotic patients treated with large doses of testosterone (Fearnley and Chakrabarti, 1962). The fibrinolytic response remained in the high physiological range, not exceeding that encountered in a minority of healthy people. It should be noted that A.C.T.H. has been reported to reduce fibrinolytic activity of pathological degree in hepatic cirrhosis (Kwaan, McFadzean, and Cook, 1956). Although the method of measurement is sensitive mainly to blood activator it does not tell us with certainty whether the change in fibrinolytic activity was due to alteration of activator or inhibitor levels. This we hope to investigate by appropriate techniques.

The rapid response of fibrinolytic activity shown by some of the patients with inflammatory disease suggested that this was a primary and specific effect of corticosteroids rather than one secondary to improvement of the inflammation. The results obtained in the five patients with non-inflammatory conditions support this, and it should be noted that, since the plasma fibrinogen levels of these patients were not raised and fell very little during treatment, the quickening of their lysistimes cannot be ascribed to change in substrate concentration. Changes in fibrinogen level in our experience have little discernible effect on blood fibrinolytic activity.

We believe it timely to regard fibrinolysis as a system of the body whose significance transcends the narrow confines of thrombolytic therapy, and, furthermore, one which is in an active state. The presence of fibrinolytic activators in body fluids besides the blood and in most of the tissues (Astrup, 1959) argues a system of general importance whose functions extend beyond the cardiovascular system.

The close association of fibrinolytic factors with fibrin and of fibrin with inflammation makes a connexion between fibrinolysis and inflammation inherently probable. The syllogism is in fact supported by a good deal of circumstantial evidence, and topical fibrinolytic therapy has been used successfully to heal chronic sinuses and ulcers (see von Kaulla, 1963). The pronounced effect of corticosteroids on blood fibrinolytic activity may be a component of their antiinflammatory action, the mechanism of which is largely obscure. A capacity to dissolve fibrin, while detrimental in peptic ulceration, wound-healing, and tuberculosis, could be beneficial in many chronic inflammatory conditions. That the influence of corticosteroids on fibrinolysis may play an 
important part in their pharmacological effects receives additional support from the incidence of low fibrinolytic activity in patients with rheumatoid arthritis and other inflammatory conditions.

\section{Summary}

A pilot study of blood fibrinolytic activity in rheumatoid arthritis led to the investigation of the effect of corticosteroid therapy on fibrinolysis.

In 17 in-patients the dilute blood-clot lysis-time, plasma fibrinogen level, and blood sedimentation rate were studied in the fasting, resting state before treatment and during treatment with corticosteroids. An increase of blood fibrinolytic activity occurred in 11 of 12 patients with inflammatory conditions and in all of 5 patients with non inflammatory conditions during treatment.

The results indicate that enhancement of fibrinolysis is a specific effect of corticosteroids, and not a consequence of improvement of inflammation.
The bearing of this finding on the therapeutic action of corticosteroids is discussed; and it is suggested that the time has come to consider fibrinolysis as a system of the body whose functions extend beyond the cardiovascular system to the tissues.

We thank Dr. R. F. Jarrett for allowing us to study two of the patients with ulcerative colitis, and Mr. John Evans for technical assistance. This study was supported by a grant from the SouthWestern Regional Hospital Board, for whose continuing support we are most grateful.

\section{REFERENCES}

Astrup, T. (1959). In Connective Tissues, Thrombosis, and Atherosclerosis, edited by I. H. Page, p. 223. Academic Press, New York and London.

Fearnley, G. R. (1951a). Proceedings of the Second A.C.T.H. Conference, New York, 11, 561 . (1951b). Lancet, 2, 501.

Balmforth, G., and Fearnley, E. (1957). Clin. Sci., 16, 645. and Bunim, J. J. (1951). Lancet, 2, 1113. and Chakrabarti, R. (1962). Ibid., 2,128 and Avis, P. R. D. (1963). Brit. med. 7., 1, 921.

and Vincent, C. T. (1960). Lancet, 2, 622

C. McFadzean, A. J. S., and Cook, J. (1956). Ibid., 1, 132

von Kaulla, K. N. (1963). Chemistry of Thrombolysis: Human Fibrinolytic Enzymes, p. 168. Thomas, Springfield, IIl., U.S.A.

\title{
Parainfluenza Infections in the Community
}

\author{
J. E. BANATVALA,* M.B., B.CHIR., D.C.H., D.P.H. ; T. B. ANDERSON, $†$ M.D. \\ B. B. REISS, $†$ M.B., B.S., M.R.C.P.
}

Brit. med.F., 1964, 1, 537-530

Since the isolation of a parainfluenza virus (Chanock, 1956) numerous investigators have demonstrated that these agents have a role in the aetiology of human respiratory infection. However, most surveys have involved select and conveniently accessible groups, such as children in hospital or residential institutions, students, or military personnel. The results of these surveys may not directly apply to members of the community living in their own homes.

We have studied the role of viruses in acute respiratory infection from September 1962 to August 1963 in the patients of two general practices in Cambridge. The complete findings will be published elsewhere, but the present paper describes our experience with respiratory infections by parainfluenza viruses. Most infection was associated with parainfluenza 1 virus, ${ }^{1}$ but a few cases of infection with parainfluenza 2 and 3 viruses also occurred.

\section{Study Population}

The material studied consisted of the patients of two general practitioners (T.B.A. and B.B.R.) working in different parts of Cambridge. The combined total was 5,178 patients and provided a cross-section of the permanent community of Cambridge-that is, the population apart from students. Patients

* Polio Research Fund Fellow, Department of Pathology, Tennis Court Road, Cambridge.

+ General Practitioner, Cambridge.

The parainfluenza viruses were originally named haemadsorption (H.A.) virus 1, croup-associated (C.A.) virus, and haemadsorption (H.A.) virus 2 , as tissue culture infected with these viruses adsorbed erythrocytes on to the cell surface. Later these viruses were renamed (Andrewes et al., 1959) myxovirus parainfluenza 1 (H.A.2), myxovirus parainfluenza 2 (C.A.), and myxovirus parainfluenza 3 (H.A.1). Sendai virus is closely related to, and represents a subtype of, parainfluenza 1 virus, although evidence of its isolation from human infection is equivocal. with acute febrile respiratory illness who were seen within 72 hours of onset were immediately referred for laboratory investigation by the general practitioner.

\section{Methods}

Collection of Specimens.-On notification of a case, one of us (J.E. B.) would visit the patient at home and obtain specimens for virological investigation. A pharyngeal swab was taken and immersed in 4-5 ml. of medium 199 containing 500 units of penicillin per ml., $500 \mu \mathrm{g}$. of streptomycin per ml., and $8 \mu \mathrm{g}$. of amphotericin B per ml. It was transported to the laboratory on crushed ice. Blood $(10 \mathrm{ml}$.) was taken at the same time from adult patients. Two to three weeks later more blood was taken and additional information about the course of the illness and the subsequent spread of the infection within the family was obtained. Occasionally specimens were collected from secondary cases in the same family, although their illnesses were not severe enough to require medical attention.

Tissue Culture.-Pharyngeal swab fluid $(0.2 \mathrm{ml}$.) was inoculated into secondary rhesus-monkey-kidney cultures within two hours of collection, the cultures having been thoroughly washed to remove all traces of calf serum. The monolayers were grown in 199 medium containing $5 \%$ calf serum, 100 units of penicillin per ml., $100 \mu \mathrm{g}$. of streptomycin per ml., $1.6 \mu$ g. of amphotericin B per ml., and $0.02 \%$ S.V.5 antiserum. Maintenance medium was similar to growth medium except that calf serum was not added. The cultures were incubated at $35^{\circ}$ C. in stationary racks, $20 \%$ of cultures being kept as uninoculated controls.

Identification of Viruses.-Cultures were tested for haemadsorption on the third, fifth, tenth, and subsequent days of incubation (Vogel and Shelokov, 1957). Fluid from cul- 